\title{
CLIFFORD ISOMETRIES \\ OF THE REAL STIEFEL MANIFOLDS
}

\author{
OSCAR A. CÁMPOLI
}

\begin{abstract}
We give a complete characterization of the elements in $S O(n)$ that are Clifford isometries of the real Stiefel manifold $S(n, k)$ of orthonormal $k$-frames in euclidean space $\mathbf{R}^{n}$.
\end{abstract}

Introduction. We will denote by $S(n, k)$ the real Stiefel manifold of orthonormal $k$-frames in $\mathbf{R}^{n}$. When we think of an element in $S(n, k)$ as a matrix of $n$ rows and $k$ columns we have a natural action of $O(n)$ on $S(n, k)$ by matrix multiplication on the left.

The restriction of that action to $S O(n)$ is transitive on $S(n, k)$ and the isotropy subgroup at the point given by the first $k$ elements of the canonical basis of $\mathbf{R}^{n}$ is the group $S O(n-k)$ included in $S O(n)$ as the subgroup with $k$ entries equal to 1 in the $k$ first diagonal places. We now have an identification

$$
S(n, k)=S O(n) / S O(n-k)
$$

and we give $S(n, k)$ an $S O(n)$ invariant normal metric.

We recall that a Clifford isometry of a metric space $(M, d)$ is an isometry $f: M \rightarrow M$ such that the displacement function $d(x, f(x))$ of $f$ is a constant function on $M$. We refer to [1] for an introduction to the general problem and the computations that follow. They give a complete characterization of the elements of $S O(n)$ that are Clifford isometries of $S(n, k)$.

The case of $S(n, 1)$ is the case of the spheres and for $S(n, n)$ we have a compact connected Lie group. Their Clifford isometries have been analyzed completely by $\mathrm{J}$. Wolf $[3,4]$ and therefore we consider $S(n, k)$ only for $1<k<n$, when we have a nonsymmetric space.

Similar calculations can be carried out in many other homogeneous spaces (see e.g. Corollary 2 at the end of this paper).

The key difference between this case and the more general situation treated in [1] is the existence of elements outside the normalizer of the maximal torus of $G$ that, by the adjoint action, send an element in the maximal torus of $H$ to the orthogonal of $H$. Of course this is impossible unless we have $\operatorname{Rank}(G)-\operatorname{Rank}(H) \geqslant 1$ and $G / H$ not a sphere. We intend next to give a general setting to this situation.

Received by the editors October 24, 1984 and, in revised form, May 24, 1985.

1980 Mathematics Subject Classification. Primary 53C30, 53C20, 58B20; Secondary 22C05. 
Matrix notations. Let $X=\left(\begin{array}{cc}0 & 1 \\ -1 & 0\end{array}\right)$ and for $i=1,2, \ldots,[n / 2]$ call $X_{i}$ the $n \times n$ real matrix that has all entries equal to zero except for a $2 \times 2$ block equal to $X$ along the diagonal in the $(n-2 i+1, n-2 i+2)$ place. Here $[n / 2]=l$ denotes the biggest integer less than or equal to $n / 2$.

The set $\mathscr{B}=\left\{X_{1}, X_{2}, \ldots, X_{l}\right\}$ is an orthogonal basis for the Lie algebra of the standard maximal torus $T$ of $G=S O(n)$ and the Weyl group $W(G, T)$ contains every permutation of $\mathscr{B}$. Let us set $r=[(n-k) / 2]$ and then the set $\mathscr{B}^{\prime}=$ $\left\{X_{1}, \ldots, X_{r}\right\}$ is an orthogonal basis for the standard maximal torus $T^{\prime}$ of $H=$ $S O(n-k)$.

Finally, we set $Y=X_{1}+\cdots+X_{l}$ and we reserve the notation $e_{1}, e_{2}, \ldots, e_{n}$ for the canonical basis of $\mathbf{R}^{n}$.

Clifford isometries. The first part of the proof of the following theorem is along the lines of the argument leading to the main result in [1]. We are grateful to the referee of the present paper who carefully read [1] and who pointed out an error which however does not affect the following considerations.

THEOREM. Let $g \in G(=S O(n))$ be a Clifford isometry of $S(n, k), 1<k<n$. If $n$ is odd, then $g=\mathrm{id}$. If $n$ is even, then $g= \pm \mathrm{id}$.

Proof. Assume that the metric in $G$ is chosen so that the length of the geodesics $\mathbf{R} \ni t \rightarrow \exp \left(t X_{i}\right)$ is $2 \pi$ for $i=1,2, \ldots, l$.

Let $g \in G$ be a Clifford isometry of $S(n, k)$. Since conjugation by an element in $G$ preserves this property and the conclusion of the theorem, assume that $g \in T$.

For $\theta_{1}, \ldots, \theta_{l} \in \mathbf{R}$ write $t\left(\theta_{1}, \ldots, \theta_{l}\right)=\exp \left(\theta_{1} X_{1}+\cdots+\theta_{l} X_{l}\right)$. Say then that $g=t\left(\theta_{1}^{0}, \ldots, \theta_{l}^{0}\right),-\pi<\theta_{i}^{0} \leqslant \pi, i=1,2, \ldots, l$, and let

$$
T_{1}=\left\{t\left(0, \ldots, 0, \theta_{r+1}, \ldots, \theta_{l}\right) / \theta_{r+1}, \ldots, \theta_{l} \in \mathbf{R}\right\} .
$$

$T_{1}$ is a closed torus of $G$ of dimension $l-r, T_{1} \cap H=\{e\}$ and the Lie algebra $\mathscr{L}\left(T_{1}\right)$ of $T_{1}$ is orthogonal to the Lie algebra $\mathscr{L}(H)$ of $H$.

It follows that $T_{1}$ is isometric via the canonical projection to a closed submanifold of $M$ (injective application with isometric derivative).

Let $g_{1}=t\left(\theta_{1}^{0}, 0, \ldots, 0\right), \quad g_{2}=t\left(0, \theta_{2}^{0}, 0, \ldots, 0\right), \ldots, g_{l}=t\left(0, \ldots, 0, \theta_{l}^{0}\right)$. Since $g_{1}, \ldots, g_{r} \in H$ we have that

$$
d(H, g \cdot H)=d\left(H, g_{r+1} \cdots g_{l} \cdot H\right)
$$

and since $T_{1}$ is isometric to a closed totally geodesic submanifold of $M$ containing $H$ and $g_{r+1} \cdots g_{l} \cdot H$, it follows that this distance can be measured inside $T_{1}$ from the identity to $g_{r+1} \cdots g_{l}$.

By the election of $\theta_{r+1}^{0}, \ldots, \theta_{l}^{0}$, this last distance is $\left(\left(\theta_{r+1}^{0}\right)^{2}+\cdots+\left(\theta_{l}^{0}\right)^{2}\right)^{1 / 2}$, i.e.,

$$
d(H, g \cdot H)^{2}=\left(\theta_{r+1}\right)^{2}+\cdots+\left(\theta_{l}^{0}\right)^{2} .
$$

Say now that $x \in G$ is such that $\operatorname{Ad}(x) X_{r}=X_{r+1}$ and $\operatorname{Ad}(x) X_{j}=X_{j}$ for $j \neq r$, $r+1$ (possible by the properties of the Weyl group). Then $x g x^{-1}=$ $t\left(\theta_{1}^{0}, \ldots, \theta_{r-1}^{0}, \theta_{r+1}^{0}, \theta_{r}^{0}, \theta_{r+2}^{0}, \ldots, \theta_{l}^{0}\right)$. 
The procedure used to obtain (1) applied to

$$
d\left(x^{-1} \cdot H, g \cdot x^{-1} \cdot H\right)=d\left(H, x \cdot g \cdot x^{-1} \cdot H\right)
$$

yields

$$
d(H, g \cdot H)^{2}=d\left(H, x g x^{-1} \cdot H\right)^{2}=\left(\theta_{r}^{0}\right)^{2}+\left(\theta_{r+2}^{0}\right)^{2}+\cdots+\left(\theta_{l}^{0}\right)^{2} .
$$

From (1) and (2) it follows that $\left|\theta_{r}^{0}\right|=\left|\theta_{r+1}^{0}\right|$ and repeating the argument with different elements of the Weyl group it follows that, after conjugation by an element of $G$ if necessary, there exists $\theta \in \mathbf{R}, 0 \leqslant \theta \leqslant \pi$, such that

$$
g=\exp \left(\theta\left(\varepsilon_{1} X_{1}+\cdots+\varepsilon_{l} X_{l}\right)\right)
$$

where $\varepsilon_{i}= \pm 1$ for $i=1,2, \ldots, l$.

Since $\left(\begin{array}{cc}1 & 0 \\ 0 & -1\end{array}\right) X\left(\begin{array}{cc}1 & 0 \\ 0 & -1\end{array}\right)=-X$ and the orthogonal group $O(n)$ acts isometrically in $S(n, k)$, after conjugation by the appropriate element of $O(n)$ we may assume

$$
g=\exp \left(\theta\left(X_{1}+\cdots+X_{l}\right)\right)=\exp (\theta Y) .
$$

We analyze several cases mainly due to notational problems: $n=2 l, n-k=2 r$, $k>2$.

Call $h$ the element in $S O(n)$ given by

$$
\begin{aligned}
h \cdot e_{1} & =e_{1}, h \cdot e_{2}=e_{3}, h \cdot e_{3}=e_{5}, h \cdot e_{4}=e_{6}, \ldots, h \cdot e_{k}=e_{k+2}, \\
h \cdot e_{k+1} & =e_{2}, h \cdot e_{k+2}=e_{4}, h \cdot e_{k+3}=e_{k+3}, \ldots, h \cdot e_{n}=e_{n} .
\end{aligned}
$$

(In case $\operatorname{det}(h)=-1$, change $h \cdot e_{1}=e_{1}$ to $h \cdot e_{1}=-e_{1}$.)

As an example we write down $h$ for $n=8, k=6$ :

$$
h=\left[\begin{array}{llllllll}
1 & 0 & 0 & 0 & 0 & 0 & 0 & 0 \\
0 & 0 & 0 & 0 & 0 & 0 & 1 & 0 \\
0 & 1 & 0 & 0 & 0 & 0 & 0 & 0 \\
0 & 0 & 0 & 0 & 0 & 0 & 0 & 1 \\
0 & 0 & 1 & 0 & 0 & 0 & 0 & 0 \\
0 & 0 & 0 & 1 & 0 & 0 & 0 & 0 \\
0 & 0 & 0 & 0 & 1 & 0 & 0 & 0 \\
0 & 0 & 0 & 0 & 0 & 1 & 0 & 0
\end{array}\right] .
$$

A direct computation shows that if we set $x=h^{-1} \cdot Y \cdot h$, the element $x$ is given by

$$
\begin{aligned}
x \cdot e_{1} & =-e_{k+1}, x \cdot e_{2}=-e_{k+2}, x \cdot e_{3}=-e_{4}, x \cdot e_{4}=e_{3}, \ldots, \\
x \cdot e_{k-1} & =-e_{k}, x \cdot e_{k}=e_{k-1}, x \cdot e_{k+1}=e_{1}, x \cdot e_{k+2}=e_{2}, \\
x \cdot e_{k+3} & =-e_{k+4}, x \cdot e_{k+4}=e_{k+3}, \ldots, x \cdot e_{n-1}=-e_{n}, x \cdot e_{n}=e_{n-1} .
\end{aligned}
$$

In the case of the example $n=8, k=6$ above we now have

$$
Y=h^{-1} \cdot Y \cdot h=\left[\begin{array}{cc|cc|cc|cc}
0 & 0 & 0 & 0 & 0 & 0 & 1 & 0 \\
0 & 0 & 0 & 0 & 0 & 0 & 0 & 1 \\
\hline 0 & 0 & 0 & 1 & 0 & 0 & 0 & 0 \\
0 & 0 & -1 & 0 & 0 & 0 & 0 & 0 \\
\hline 0 & 0 & 0 & 0 & 0 & 1 & 0 & 0 \\
0 & 0 & 0 & 0 & -1 & 0 & 0 & 0 \\
\hline-1 & 0 & 0 & 0 & 0 & 0 & 0 & 0 \\
0 & -1 & 0 & 0 & 0 & 0 & 0 & 0
\end{array}\right] .
$$


As we showed in the proof of the main result in [1], we have

$$
d(H, g \cdot H)^{2}=(l-r) \theta^{2}
$$

and since $h^{-1} \cdot g \cdot h=\exp (\theta x)=\exp \left(\theta\left(h^{-1} \cdot Y \cdot h\right)\right)$, if we assume that $\theta$ is such that $\sin \theta \neq 0$, using that $g$ is a Clifford isometry we obtain

$$
(l-r) \theta^{2}=d(H, g \cdot H)^{2}=d\left(H, h^{-1} \cdot g \cdot h \cdot H\right)^{2}=(l-r+1) \theta^{2},
$$

which is impossible.

We must then have $\sin \theta=0$ and hence $g= \pm \mathrm{id}$. as we wanted to show.

In case $k=2$ we may proceed in a similar way now using the element

$$
h \cdot e_{1}=e_{1}, h \cdot e_{2}=e_{4}, h \cdot e_{3}=e_{2}, h \cdot e_{4}=e_{4}, \ldots, h \cdot e_{n}=e_{n} .
$$

On the other hand, when $n=2 l, n-k=2 r+1$ since $0<n-k<n-1$ the same calculations above apply.

Finally we should address ourselves to the case $n=2 l+1$.

The only difference we should note in this situation is that the possibility $\theta=\pi$ is ruled out due to the necessary presence of the eigenvalue 1 in $g$, which makes it impossible then to have -1 also as the eigenvalue for $g$. Q.E.D.

COROllary 1. Let $\Gamma \subset S O(n)$ be a finite group of Clifford isometries of $S(n, k)$. Then $S(n, k) / \Gamma$ is a homogeneous Riemannian manifold.

Note. In connection with this corollary see the conjecture in Wolf $[2$, p. 230] or the introduction in [1].

Corollary 2 (OF THE PROOF). Let $G=S O(2 l+1)$ and let $H$ be a closed connected Lie subgroup of $G$ that has a maximal torus that is a standard subtorus of the standard maximal torus of $G$. Then the only Clifford isometry of $M=G / H$ that $G$ contains is the identity.

\section{REFERENCES}

1. O. A. Cámpoli, Clifford isometries of compact homogeneous Riemannian manifolds, Rev. Un. Mat. Argentina 31 (1983), 44-49.

2. J. A. Wolf, Spaces of constant curvature, 4th ed., Publish or Perish, Berkeley, Calif.

3. Locally symmetric homogeneous spaces, Comm. Math. Helv. 37 (1962-63), 65-101.

4. Vincent's conjecture on Clifford translations of the sphere, Comm. Math. Helv. 36 (1962), $33-41$.

Facultad de Matemática, Astronomía y Fisica, Universidad Nacional de Córdoba, Avdas. Valparaíso y Rogelio Martínez, Ciudad Universitaria - 5000 Cordoba, Argentina 